\title{
Formation of Cone-Shaped Inclusions and Line Defects on the Cz-Si Wafer Surface by the Helium Implantation and DC Nitrogen Plasma Treatment
}

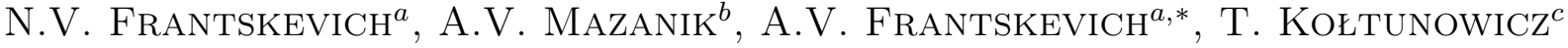 \\ AND P. ŻUKOWSKI ${ }^{c}$ \\ ${ }^{a}$ Belarussian National Technical University, 65, Nezavisimosti St., 220063 Minsk, Belarus \\ ${ }^{b}$ Belarussian State University, 4, Nezavisimosti St., 220030 Minsk, Belarus \\ ${ }^{c}$ Lublin University of Technology, Nadbystrzycka 38, 20-618 Lublin, Poland
}

\begin{abstract}
The general goal of this work is to investigate the defects formed on the surface of the Cz-Si wafers subjected to helium implantation, vacuum annealing and nitrogen plasma treatment. The performed scanning electron microscopy study has shown that in the general case two types of surface defects can be formed: cone-shaped inclusions with the base diameter of $0.2-2 \mu \mathrm{m}$ and the ratio of diameter to height of approximately $1: 1$, as well as crystallographically oriented line defects with the length equal to $0.2-2 \mu \mathrm{m}$. The concentration of these defects depends on the conditions of implantation and plasma treatment.
\end{abstract}

PACS: $61.72 . u f, 73.20 . \mathrm{Hb}$

\section{Introduction}

In work [1] we investigated the possibility of forming buried insulating $\mathrm{Si}_{x} \mathrm{~N}_{y}$ layers by a new method based on the nitrogen gettering onto the buried defect layer created by helium implantation.

The topicality of investigations presented in this work is determined by the fact that the practical appropriateness of silicon-on-insulator wafers formed by the method proposed in [1] requires the conservation of perfectness of silicon wafer surface and its near-surface (working) area. On the other hand, the plasma influence can modify the surface properties due to both introduction of impurity atoms (nitrogen, in our case) and formation of defects by ion beam. In this connection, the study of defects arising as a result of plasma treatment of preliminarily implanted wafers is of significant scientific and practical interest.

\section{Experimental}

Standard $4.5 \Omega \mathrm{cm} n$-type and $12 \Omega \mathrm{cm}$-type $(100)$ $\mathrm{Cz}-\mathrm{Si}$ wafers were subjected to helium ion implantation at room temperature with the energy of $300 \mathrm{keV}$ and the fluences of $1 \times 10^{15}, 5 \times 10^{15}, 1 \times 10^{16}$ or $2 \times 10^{16}$ at. $/ \mathrm{cm}^{2}$. After implantation the samples were annealed in vacuum at $800^{\circ} \mathrm{C}$ for $1 \mathrm{~h}$. Then nitrogen was introduced into silicon from a DC plasma source at $350^{\circ} \mathrm{C}$. Nitrogen plasma

* corresponding author; e-mail: a.frantskevich@rambler.ru treatments were conducted at two ion current densities that differ by the factor of 3 . The total nitrogen dose in all cases was equal to $4 \times 10^{16}$ at. $/ \mathrm{cm}^{2}$. The surface defects arising as a consequence of the treatments were studied by scanning electron microscopy (SEM) using LEO1455 microscope.

\section{Results and discussion}

The experiments have shown that the line and cone-shaped defects are formed on the surface of the treated silicon wafer (Figs. 1-4). The typical length of line defects on the surface of wafers subjected to plasma treatment at lower current density equals $1-2 \mu \mathrm{m}$ (Fig. 2 ). It is seen from Figs. 1 and 2 that the line defects are oriented in two inter-perpendicular directions. Inasmuch as the studied samples have (100) surface, one can assume that the observed line defects are oriented along [100]-like directions.

Along with this type of defects, the cone-shaped ones can be formed (Figs. 3 and 4). These cone-shaped inclusions can arise both jointly and separately with line defects. The base diameter of cone-shaped defects is equal to $0.2-2 \mu \mathrm{m}$, the ratio of diameter to height is approximately 1:1. The X-ray microanalysis of cone-like defects does not reveal any chemical elements except silicon and nitrogen. Such inclusions have enhanced electron emission ability in comparison with the silicon surface.

In the case of sample treatment at greater current density, the cone-shaped inclusions are formed rarely. In so 


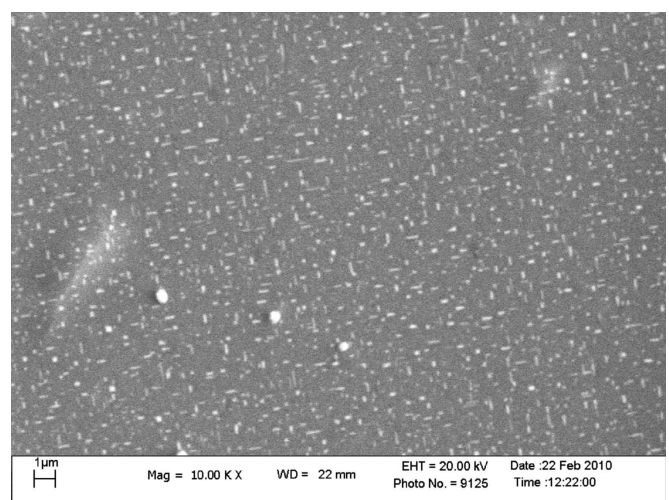

Fig. 1. SEM image of $4.5 \Omega \mathrm{cm} n$-type $\mathrm{Cz}$-Si wafer implanted by helium with a fluence of $1 \times 10^{15} \mathrm{~cm}^{-2}$, vacuum annealed at $800^{\circ} \mathrm{C}$ for $1 \mathrm{~h}$ and nitrogen plasma treated at greater current density.

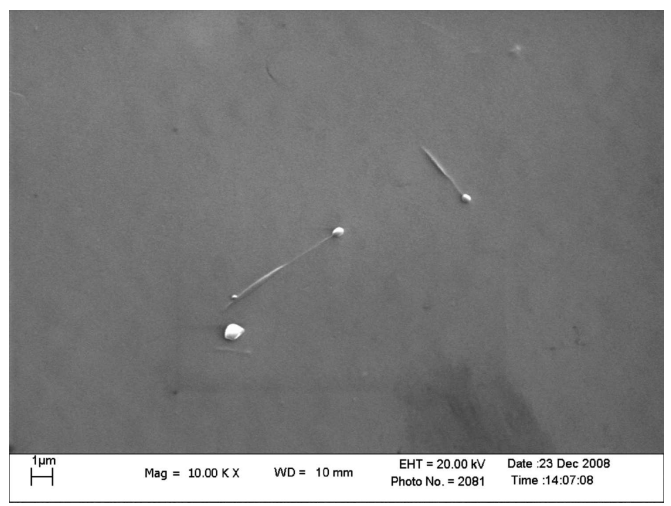

Fig. 2. SEM image of $4.5 \Omega \mathrm{cm} n$-type $\mathrm{Cz}$-Si wafer implanted by helium with a fluence of $1 \times 10^{15} \mathrm{~cm}^{-2}$, vacuum annealed at $800^{\circ} \mathrm{C}$ for $1 \mathrm{~h}$ and nitrogen plasma treated at lower current density.

doing, the surface defectness is higher for small implantation fluences and the typical length of crystallographically oriented line defects equals $200-500 \mathrm{~nm}$ (Fig. 1). The increase of helium implantation fluence leads to decrease of a number of defects and in the case of optimal conditions of ion implantation and plasma treatment the defects on the surface are absent. For lower current density such trend (decrease of surface defects density with the increase of implantation fluence) takes place too, however, no defect-free surface was observed.

Let us try to understand the origin of the observed cone-shaped inclusions. First of all, note that their formation cannot be explained by the non-uniform plasma etching of the sample surface. Indeed, according to SRIM-2008 calculations, at the used regimes of plasma treatment the thickness of plasma-etched silicon layer does not exceed a few tens of nanometers, whereas the height of the cone-shaped inclusions can reach one micrometer. As it can be seen in the SEM image presented in Fig. 5, the surface defect, which looks as a dark spot

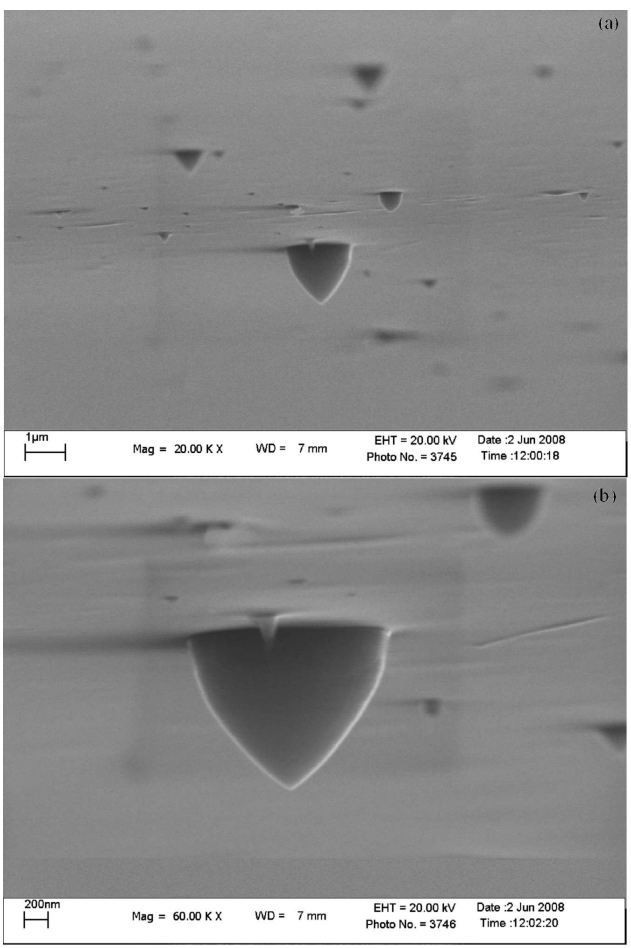

Fig. 3. SEM images with different magnification of $12 \Omega \mathrm{cm} p$-type $\mathrm{Cz}$-Si wafer implanted by helium with a fluence of $1 \times 10^{16} \mathrm{~cm}^{-2}$, vacuum annealed at $800{ }^{\circ} \mathrm{C}$ for $1 \mathrm{~h}$ and nitrogen plasma treated at lower current density.

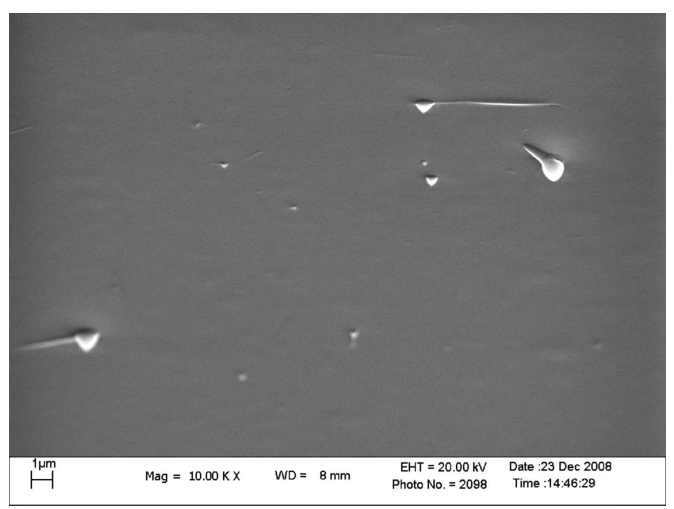

Fig. 4. SEM images of $12 \Omega \mathrm{cm}$-type $\mathrm{Cz}$-Si wafer implanted by helium with a fluence of $1 \times 10^{15} \mathrm{~cm}^{-2}$, vacuum annealed at $800^{\circ} \mathrm{C}$ for $1 \mathrm{~h}$ and nitrogen plasma treated at lower current density.

at the image center and acts as the source of mechanical stresses, is surrounded by cone-shaped inclusions. This result makes it possible to presume that the formation of the observed cone-shape inclusions is related to the presence of mechanical stresses in the near-surface region of wafer. According to [2], the annealing of the wafers implanted by light ions (hydrogen, helium) can significantly modify the structure of the $R_{p}$ thick near-surface region 


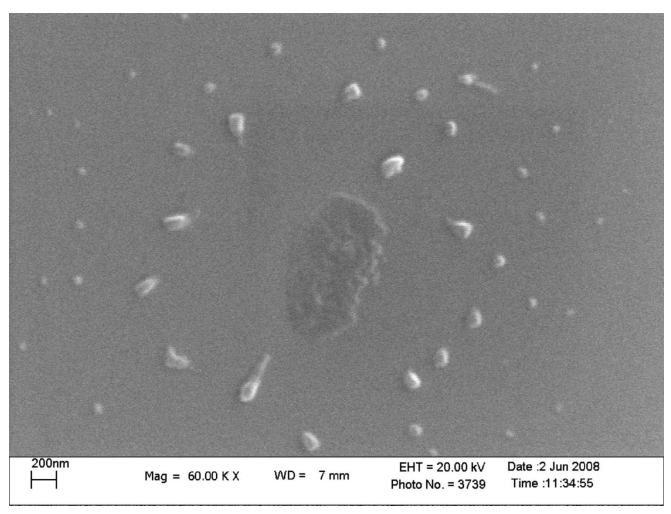

Fig. 5. SEM image of $4.5 \Omega \mathrm{cm} n$-type $\mathrm{Cz}$-Si wafer implanted by helium with a fluence of $1 \times 10^{16} \mathrm{~cm}^{-2}$, vacuum annealed at $800^{\circ} \mathrm{C}$ for $1 \mathrm{~h}$ and nitrogen plasma treated at lower current density.

due to the development of the buried defect layer created by implantation. In particular, a system of defects normal to the surface may be formed due to the propagation of defects created in the buried helium-containing layer [3]. One can conclude that the formation of the observed cone-shaped inclusions as a result of introduction of nitrogen from the plasma is determined by the presence of the defects and mechanical stresses in the near-surface wafer region.

\section{Conclusion}

1. The formation of a new type of defects on the surface of silicon wafers subjected to helium implantation, annealing and nitrogen plasma treatment was observed. These defects have a cone-like shape with the base diameter of 200-2000 $\mathrm{nm}$ and the ratio of diameter to height of approximately 1:1.

2. Along with the cone-shaped inclusions, the crystallographically oriented line defects are formed. The concentrations of both types of defects depend on the relation of implantation and plasma treatment regimes. The optimal choice of these conditions allows to obtain the defect-free silicon surface.

\section{References}

[1] N.V. Frantskevich, A.V. Frantskevich, A.K. Fedotov, A.V. Mazanik, Solid State Phenomena 156-158, 91 (2010).

[2] B. Terreault, Physica Status Solidi A 204, 2129 (2007).

[3] A.V. Frantskevich, A.M. Saad, A.V. Mazanik, N.V. Frantskevich, A.K. Fedotov, V.S. Kulinkauskas, A.A. Patryn, J. Mater. Sci. Mater. Electron. 19 S239 (2008). 